\title{
Time-Reversal Space-Time Coding for Doubly-Selective Channels
}

\author{
Stefan Geirhofer, Lang Tong, and Anna Scaglione \\ School of Electrical and Computer Engineering \\ Cornell University, Ithaca, NY 14853 \\ Email: $\{\operatorname{sg} 355,1 \mathrm{t} 35$, as337\}@ cornell.edu
}

\begin{abstract}
We consider the transmission of space-time block codes (STBC) over doubly-selective channels, which are encountered in highly mobile environments or high-frequency (HF) communications. In particular, we propose two extensions of time-reversal STBC with superior performance under these fast-fading conditions, and compare these schemes for various decision-feedback equalizers. The tradeoffs between performance and complexity are illustrated, and simulation results for the severely doubly-selective HF-channel corroborate our analysis.
\end{abstract}

\section{INTRODUCTION}

Space-time block codes (STBC) are an attractive means for gaining diversity in wireless communications due to their promising performance yet simplicity in implementation. While many contributions exist on how to extend Alamouti's scheme [1] to the frequency-selective case, the channel's fading characteristics have received little attention. However, taking time-selectivity into consideration will become indispensable in future cellular systems, where high-mobility applications are a major concern.

\section{A. Existing methods}

Alamouti's seminal scheme [1] has received enormous attention in the literature. While the original proposal is restricted to flat-fading channels, many contributions have been made on how to extend this idea to the frequencyselective case. In particular, Time-Reversal STBC [2], Orthogonal Frequency Division Multiplexing (OFDM) [3], and Single-Carrier Frequency Domain Equalization (SC-FDE) [4], [5] have received the most attention and are compared in [6] for the Typically-Urban (TU) GSM channel model.

Both OFDM and SC-FDE rely on the transmission of a cyclic prefix, which renders the channel matrices circulant. This property is important since it allows for effective equalization in the frequency domain by diagonalizing the matrices via the FFT. On the contrary, TR-STBC operates in the time-domain applying Alamouti's ideas on blocks instead of symbols. Spatio-temporal matched filtering then transforms the receive processing into conventional block decoding while preserving full diversity gain and allowing perfect decoupling between the blocks [2], [6]. The approaches mentioned so far, however, crucially rely on the channel being constant over the transmission of an entire space-time codeword. While this assumption is justified in some scenarios, it is inappropriate for high-mobility applications, or the HF-channel which is severely doubly-selective.

\footnotetext{
${ }^{1}$ This work was supported under the ONR Grant N00014-00-1-0564.
}

Some propositions have been made on how to exploit diversity in doubly selective channels [7], [8], relying on basis expansion models (BEM) [9] to exploit both space and time diversity. While full diversity can be achieved with such techniques, extensive precoding is necessary making it difficult to apply these methods in a block transmission system.

In this paper we will consider the HF-channel as a representative example of a severely doubly-selective channel. In this area, space-time techniques face the fundamental problem that large antenna spacings are required due to the low frequency location of the HF band. Even though it might thus be impractical to integrate multiple antennas into one terminal, our novel designs could be widely applied to distributed space-time coding systems [10] and contribute to significantly improving the transmission rate.

\section{B. This work's contribution}

This paper proposes two extensions of TR-STBC that show superior performance in a doubly selective channel. In particular, we consider shortening the data block length and/or extending the matched filtering to the varying channel. A timedomain approach is favored because it can easily be adapted to block transmission systems with alternating pilot and data intervals. Our results bolster four important conclusions: (i) TR-STBC is sensitive to channel variations, (ii) our schemes are robust for reasonable Doppler spreads while still allowing for efficient implementations (Toeplitz structure is retained), (iii) the performance loss compared to a symbol-by-symbol characterization of the channel is small, (iv) while one of the proposed methods (MF-method) achieves satisfactory performance with conventional decision-feedback equalization, the other (SB-method) requires the use of nonlinear data-directed estimation (NDDE).

The rest of the paper is organized as follows. In Sec. II we describe our proposed extensions of TR-STBC. Subsequently, Sec. III illustrates how decision-feedback equalization and nonlinear data-directed estimation can be used to perform block-decoding. The channel model as well as simulation parameters are outlined in Sec. IV keeping compatibility with HF-standards [11] (and references therein) in focus. Detailed simulation results corroborating our analysis are presented in Sec. V, and a summary concludes this work.

Notation: Bold uppercase (lowercase) letters denote matrices (column vectors). Hermitian transpose is written $(\cdot)^{H}$. TimeReversal is denoted by adding tilde $(\tilde{\cdot})$ and written mathematically as $\tilde{\mathbf{a}}=\mathbf{J a}$ for a vector $\mathbf{a}$ and $\tilde{\mathbf{A}}=\mathbf{J A J}$ for a matrix 


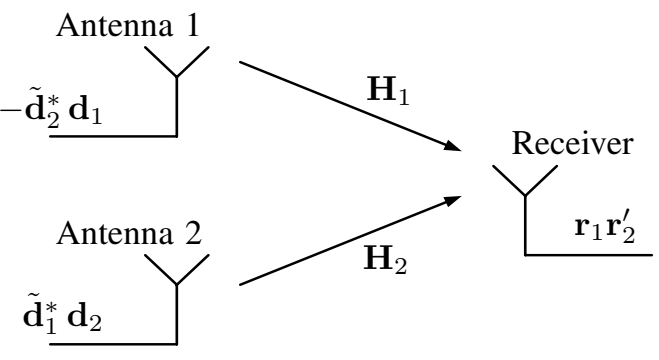

Fig. 1. Block diagram of the antenna configuration.

$\mathbf{A}$, where $\mathbf{J}$ denotes the counteridentity matrix, i.e. a matrix with ones on the secondary diagonal and zeros elsewhere.

\section{Algorithms}

In this section we first briefly review TR-STBC [2] and then discuss our extensions, which deal with the time-varying channel. In the performance assessment the channel is continuously varying from symbol to symbol. However, for the sake of finding computationally efficient algorithms the proposed schemes assume a constant channel over sufficiently short periods of time. In our mathematical analysis we can thus still consider Toeplitz (instead of banded) matrices.

\section{A. Time-Reversal STBC}

We start by introducing TR-STBC, whose operation is depicted in Fig. 1. At the transmitter the source block $\mathbf{d}$ is split up in $\mathbf{d}_{1}$ and $\mathbf{d}_{2}$ which are both blocks of length $N$. Now, consider the following transmission scheme: at first $\mathbf{d}_{1}$ is transmitted from the first antenna and $\mathbf{d}_{2}$ from the second. At the receiver we obtain

$$
\mathbf{r}_{1}=\mathbf{H}_{1} \mathbf{d}_{1}+\mathbf{H}_{2} \mathbf{d}_{2}+\mathbf{n}_{1}
$$

where $\mathbf{r}_{1}$ is the received block of $N$ samples, $\mathbf{H}_{i}$ model the convolution with the channels and $\mathbf{n}_{1}$ is additive white Gaussian noise. In the second transmission period we transmit $-\tilde{\mathbf{d}}_{2}^{*}$ from the first antenna and $\tilde{\mathbf{d}}_{1}^{*}$ from the second. We thus receive

$$
\tilde{\mathbf{r}}_{2}^{\prime}=-\mathbf{H}_{1} \tilde{\mathbf{d}}_{2}^{*}+\mathbf{H}_{2} \tilde{\mathbf{d}}_{1}^{*}+\mathbf{n}_{2}^{\prime}
$$

which is time-reversed and conjugated at the receiver to form

$$
\mathbf{r}_{2}=\mathbf{J}_{2}^{\prime}=\tilde{\mathbf{H}}_{2}^{*} \mathbf{d}_{1}-\tilde{\mathbf{H}}_{1}^{*} \mathbf{d}_{2}+\mathbf{n}_{2}
$$

It should be noted that some type of guard interval is necessary to avoid inter-block interference between $\mathbf{r}_{1}$ and $\mathbf{r}_{2}$. We can now rewrite (1) and (3) as

$$
\underbrace{\left[\begin{array}{c}
\mathbf{r}_{1} \\
\mathbf{r}_{2}
\end{array}\right]}_{\mathbf{r}}=\underbrace{\left[\begin{array}{cc}
\mathbf{H}_{1} & \mathbf{H}_{2} \\
\tilde{\mathbf{H}}_{2}^{*} & -\tilde{\mathbf{H}}_{1}^{*}
\end{array}\right]}_{\mathbf{H}} \underbrace{\left[\begin{array}{c}
\mathbf{d}_{1} \\
\mathbf{d}_{2}
\end{array}\right]}_{\mathbf{d}}+\underbrace{\left[\begin{array}{c}
\mathbf{n}_{1} \\
\mathbf{n}_{2}
\end{array}\right]}_{\mathbf{n}}
$$

At the receiver, $\mathbf{r}$ is processed with the spatio-temporal matched filter $\mathbf{H}^{H}$

$$
\mathbf{y}=\mathbf{H}^{H} \mathbf{r}=\mathbf{H}^{H} \mathbf{H d}+\mathbf{H}^{H} \mathbf{n}
$$

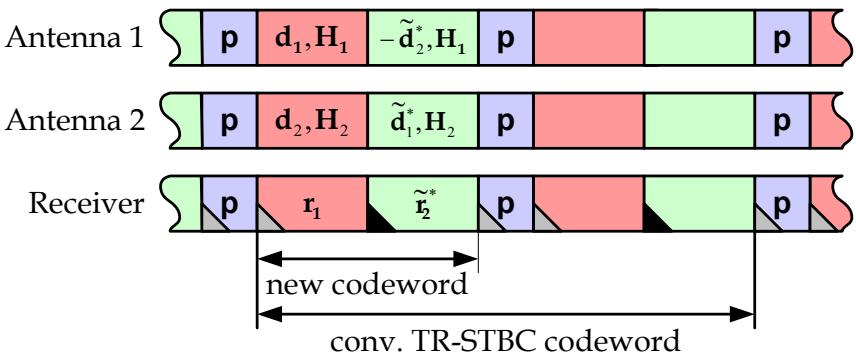

Fig. 2. Timing diagram for the short-block approach (IBI is denoted by shaded triangles). The new codeword is half the length of conventional TRSTBC, but shows some IBI between both blocks.

which perfectly decouples the decoding of the blocks $\mathbf{d}_{1}$ and $\mathbf{d}_{2}$ since all off-diagonal terms of $\mathbf{H}^{H} \mathbf{H}$ are zero,

$$
\mathbf{H}^{H} \mathbf{H}=\left[\begin{array}{cc}
\mathbf{H}_{1}^{H} \mathbf{H}_{1}+\mathbf{H}_{2}^{H} \mathbf{H}_{2} & \mathbf{0} \\
\mathbf{0} & \mathbf{H}_{1}^{H} \mathbf{H}_{1}+\mathbf{H}_{2}^{H} \mathbf{H}_{2}
\end{array}\right] .
$$

It can easily be shown that the covariance matrix of the noise $\mathbf{H}^{H} \mathbf{n}$ is also given by a scaled version of (6). Thus the decoding of $\mathbf{d}_{1}$ and $\mathbf{d}_{2}$ fully decouples.

While TR-STBC is an elegant means to cope with frequency-selective channels, it makes two fundamental assumptions. First, it requires the insertion of guard intervals of greater length than the memory of the channel in order to avoid inter-block interference at the receiver. Secondly, the channel must be constant in order for the $\mathbf{H}_{i}$ s to be Toeplitz and the above properties to hold. Our results show that TR-STBC's performance worsens drastically if this condition is violated.

\section{B. Short-Block Approach}

The time-variation within one block can be mitigated by shortening the block length. It should be noted however that while the block-length of the data can be (in theory) arbitrarily chosen, this does not hold true for the guard interval, since its length is lower-bounded by the length of the channel.

For this reason, we propose to shrink the data block size by a factor of two, while not inserting a guard interval between adjacent blocks The lack of a guard interval results in interblock interference (IBI) between the blocks, which needs to be removed later as depicted in Fig. 2.

Mathematically, we can express the received signal during the first half of the transmission as

$$
\mathbf{r}_{1}=\mathbf{H}_{1}\left[\begin{array}{c}
\mathbf{p} \\
\mathbf{d}_{1} \\
-\tilde{\mathbf{d}}_{2 e}^{*}
\end{array}\right]+\mathbf{H}_{2}\left[\begin{array}{c}
\mathbf{p} \\
\mathbf{d}_{2} \\
\tilde{\mathbf{d}}_{1 e}^{*}
\end{array}\right]+\mathbf{n}_{1},
$$

where $\mathbf{H}_{1}$ and $\mathbf{H}_{2}$ are now modeled as Toeplitz matrices with size $(N+L-1) \times(N+2 L-2)$ (we have to take IBI into account), $\mathbf{r}_{1}$ is a vector of length $N+L-1$ and $\mathbf{p}$ denotes the (known) probe symbols. The symbols $\mathbf{d}_{1 e}$ and $\mathbf{d}_{2 e}$ denote vectors of the last $L-1$ symbols of each block. Similarly, we get for the second block after conjugation and time-reversal at 
the receiver

$$
\mathbf{r}_{2}=\tilde{\mathbf{H}}_{1}^{*}\left[\begin{array}{c}
\tilde{\mathbf{p}}^{*} \\
-\mathbf{d}_{2} \\
\tilde{\mathbf{d}}_{1 e}^{*}
\end{array}\right]+\tilde{\mathbf{H}}_{2}^{*}\left[\begin{array}{c}
\tilde{\mathbf{p}}^{*} \\
\mathbf{d}_{1} \\
\tilde{\mathbf{d}}_{2 e}^{*}
\end{array}\right]+\tilde{\mathbf{n}}_{2}^{*} .
$$

The first step in processing this signal is to remove the interference caused by the pilot symbols. Since both these and the channel are assumed perfectly known this amounts to a simple subtraction. Denoting this vector by $\mathbf{r}^{\prime}$ we find

$$
\begin{aligned}
& \underbrace{\left[\begin{array}{l}
\mathbf{r}_{1}^{\prime} \\
\mathbf{r}_{2}^{\prime}
\end{array}\right]}_{\mathbf{r}^{\prime}}= \underbrace{\left[\begin{array}{cc}
\mathbf{H}_{1 m} & \mathbf{H}_{2 m} \\
\tilde{\mathbf{H}}_{2 m}^{*} & -\tilde{\mathbf{H}}_{1 m}^{*}
\end{array}\right]}_{\mathbf{H}_{s}} \underbrace{\left[\begin{array}{l}
\mathbf{d}_{1} \\
\mathbf{d}_{2}
\end{array}\right]}_{\mathbf{d}}+ \\
& \underbrace{\left[\begin{array}{cc}
\mathbf{H}_{2 t} & -\mathbf{H}_{1 t} \\
\tilde{\mathbf{H}}_{1 t}^{*} & \tilde{\mathbf{H}}_{2 t}^{*}
\end{array}\right]}_{\mathbf{H}_{i}}\left[\begin{array}{c}
\mathbf{d}_{1 e}^{*} \\
\mathbf{d}_{2 e}^{*}
\end{array}\right]+\underbrace{\left[\begin{array}{l}
\mathbf{n}_{1} \\
\mathbf{n}_{2}
\end{array}\right]}_{\mathbf{n}},
\end{aligned}
$$

where $\mathbf{H}_{1 m}, \mathbf{H}_{2 m}$ denote the $N$ center columns and $\mathbf{H}_{1 t}, \mathbf{H}_{2 t}$ consist of the last $L-1$ columns of $\mathbf{H}_{1}$ and $\mathbf{H}_{2}$, respectively.

As a result of the inter-block interference we cannot expect the decoding of $\mathbf{d}_{1}$ and $\mathbf{d}_{2}$ to decouple fully at the receiver. However we can solve the above equation for $\mathbf{d}$ jointly. In order to do so we need to rewrite the conjugated terms on the right hand side of (9). This can be done in real form by considering the following transformation for vectors $\mathbf{z}$ and matrices A respectively [12],

$$
\hat{\mathbf{z}}=\left[\begin{array}{c}
\mathfrak{R e}(\mathbf{z}) \\
\mathfrak{I m}(\mathbf{z})
\end{array}\right] \quad \hat{\mathbf{A}} \rightarrow\left[\begin{array}{cc}
\mathfrak{R e}(\mathbf{A}) & -\mathfrak{I m}(\mathbf{A}) \\
\mathfrak{I m}(\mathbf{A}) & \mathfrak{R e}(\mathbf{A})
\end{array}\right] .
$$

It is proved in [12] that for the above transformation the following properties hold

$$
\begin{aligned}
\mathbf{C}=\mathbf{A B} & \Leftrightarrow \hat{\mathbf{C}}=\hat{\mathbf{A}} \hat{\mathbf{B}} \\
\mathbf{C}=\mathbf{A}+\mathbf{B} & \Leftrightarrow \hat{\mathbf{C}}=\hat{\mathbf{A}}+\hat{\mathbf{B}} \\
\mathbf{C}=\mathbf{A}^{H} & \Leftrightarrow \hat{\mathbf{C}}=\hat{\mathbf{A}}^{T} \\
\mathbf{C}=\mathbf{A}^{-1} & \Leftrightarrow \hat{\mathbf{C}}=\hat{\mathbf{A}}^{-1}
\end{aligned}
$$

where $\hat{\mathbf{B}}, \hat{\mathbf{C}}$ are defined in the same way as $\hat{\mathbf{A}}$. Thus we get

$$
\begin{gathered}
{\left[\begin{array}{c}
\mathfrak{R e}\left(\mathbf{r}^{\prime}\right) \\
\mathfrak{I m}\left(\mathbf{r}^{\prime}\right)
\end{array}\right]=\left[\begin{array}{cc}
\mathfrak{R e}\left(\mathbf{H}_{s}\right) & -\mathfrak{I m}\left(\mathbf{H}_{s}\right) \\
\mathfrak{I m}\left(\mathbf{H}_{s}\right) & \mathfrak{R e}\left(\mathbf{H}_{s}\right)
\end{array}\right]\left[\begin{array}{c}
\mathfrak{R e}(\mathbf{d}) \\
\mathfrak{I m}(\mathbf{d})
\end{array}\right]+} \\
{\left[\begin{array}{cc}
\mathfrak{R e}\left(\mathbf{H}_{i}\right) & \mathfrak{I m}\left(\mathbf{H}_{i}\right) \\
\mathfrak{I m}\left(\mathbf{H}_{i}\right) & -\mathfrak{R e}\left(\mathbf{H}_{i}\right)
\end{array}\right]\left[\begin{array}{c}
\mathfrak{R e}\left(\mathbf{d}_{e}\right) \\
\mathfrak{I m}\left(\mathbf{d}_{e}\right)
\end{array}\right]+\left[\begin{array}{c}
\mathfrak{R e}(\mathbf{n}) \\
\mathfrak{I m}(\mathbf{n})
\end{array}\right] .}
\end{gathered}
$$

Finally, we can simplify this equation and write

$$
\underline{\mathbf{r}}^{\prime}=\underline{\mathbf{H}} \underline{\mathbf{d}}+\underline{\mathbf{n}},
$$

where the underlined symbols are now real vectors and matrices of twice the size as before. A spatio-temporal matched filter now concludes this proposed scheme

$$
\underline{\mathbf{y}}=\underline{\mathbf{H}}^{T} \underline{\mathbf{H}} \underline{\mathbf{d}}+\underline{\mathbf{H}}^{T} \underline{\mathbf{n}}=\underline{\mathbf{R}} \underline{\mathbf{d}}+\underline{\mathbf{v}} .
$$

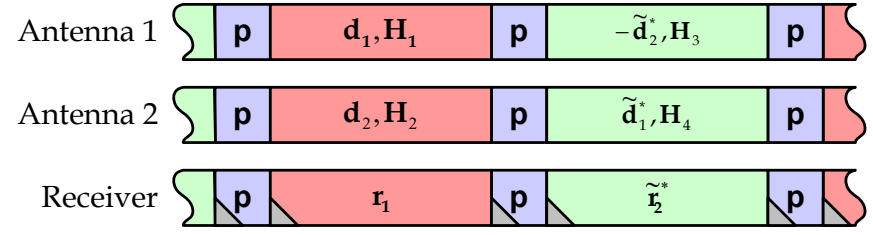

Fig. 3. Timing diagram for the matched-filter approach

\section{Matched-Filter Approach}

Another possible approach is not to reduce the block size, but to better approximate the continuously varying channel by matrices $\mathbf{H}_{1}$ through $\mathbf{H}_{4}$ as illustrated in Fig. 3. Essentially, this corresponds to extending the matched-filtering approach to the varying channel.

We thus arrive at

$$
\mathbf{r}_{1}=\mathbf{H}_{1} \mathbf{d}_{1}+\mathbf{H}_{2} \mathbf{d}_{2}+\mathbf{n}_{1}
$$

during the first transmission period, and

$$
\mathbf{r}_{2}^{\prime}=-\mathbf{H}_{3} \tilde{\mathbf{d}}_{2}^{*}+\mathbf{H}_{4} \tilde{\mathbf{d}}_{1}^{*}+\mathbf{n}_{2}^{\prime}
$$

during the second. Conjugation and time reversal of the second equation and rewriting in matrix form gives

$$
\left[\begin{array}{l}
\mathbf{r}_{1} \\
\mathbf{r}_{2}
\end{array}\right]=\left[\begin{array}{cc}
\mathbf{H}_{1} & \mathbf{H}_{2} \\
\tilde{\mathbf{H}}_{4}^{*} & -\tilde{\mathbf{H}}_{3}^{*}
\end{array}\right]\left[\begin{array}{l}
\mathbf{d}_{1} \\
\mathbf{d}_{2}
\end{array}\right]+\left[\begin{array}{l}
\mathbf{n}_{1} \\
\mathbf{n}_{2}
\end{array}\right] .
$$

If we now again employ spatio-temporal matched filtering we get

$$
\mathbf{y}=\left[\begin{array}{cc}
\mathbf{H}_{1}^{H} \mathbf{H}_{1}+\mathbf{H}_{3}^{H} \mathbf{H}_{3} & \mathbf{H}_{1}^{H} \mathbf{H}_{3}-\mathbf{H}_{2}^{H} \mathbf{H}_{4} \\
\mathbf{H}_{1}^{H} \mathbf{H}_{3}-\mathbf{H}_{2}^{H} \mathbf{H}_{4} & \mathbf{H}_{2}^{H} \mathbf{H}_{2}+\mathbf{H}_{4}^{H} \mathbf{H}_{4}
\end{array}\right] \mathbf{d}+\mathbf{n},
$$

which can be decoded in complex form by employing the methods described in the next section.

\section{Block-Decoding Algorithms}

The space-time coding schemes considered in the last section transform the decoding process into a conventional block decoding problem. The original proposal of TR-STBC [2] suggests a whitening filter followed by a maximum likelihood sequence estimator. In the scope of this paper we shall, apart from standard ZF and MMSE decoders, explore a different approach, namely the use of decision-feedback structures. In particular, we will consider block decision feedback equalization [13], as well as nonlinear data-directed estimation (NDDE), a scheme specifically devised for HF-communications [14].

The performance of linear ZF and MMSE equalization is considerably outperformed by decision-feedback techniques. This stems from the fact that data symbols close to the block ends have smaller variance than symbols in the middle of a block [14]. This is intuitive for two reasons. First, the channel is estimated by employing the probe symbols and this estimate is best close to the block ends. Secondly, the ISI resulting from the probe data can easily be suppressed, again facilitating decoding at the block ends. 


\section{A. ZF/MMSE Decision Feedback Equalization}

We first deal with ZF and MMSE block decision feedback equalization [13]. The equalizer consists of a feed-forward noise-whitening filter as well as a feedback filter that presumably feeds back the correct decisions. The coefficients for both filters can be found by Cholesky factorization of the covariance matrix $\mathbf{R}$. The decoding is then performed starting from the end of the block (see [13] for detailed treatment).

\section{B. Nonlinear Data-Directed Estimation}

The nonlinear data-directed estimator (NDDE) [14] is specifically tailored to the severely doubly-selective HFchannel. It outperforms BDFE outlined above, since it decodes from both block ends (where the symbol variance is the least) towards the middle of the block. This is especially useful in the short-block approach, since errors made in the IBI region cannot propagate any further.

According to the above we start with

$$
\hat{\mathbf{d}}^{(1)}=\left(\mathbf{R}+\frac{2 N_{0}}{\sigma_{d}^{2}} \mathbf{I}\right)^{-1} \mathbf{y},
$$

the linear MMSE solution. However, according to our assumption we only keep the first and the last symbol in this vector. Subsequently we subtract these symbols' interference

$$
\mathbf{y}^{(2)}=\mathbf{y}-[\mathbf{R}]_{1} d_{1}-[\mathbf{R}]_{N} d_{N},
$$

where $[\mathbf{R}]_{i}$ denotes the $i$-th column of $\mathbf{R}$. We then again perform linear MMSE equalization, and iteratively follow this procedure until all symbols are decoded.

\section{Simulation Model}

In this section an overview of Watterson's channel model is given and the simulation parameters are outlined.

\section{A. Channel Model}

The accurate modeling of the HF-channel plays a crucial role in our analysis, since it determines to what extent the channel can be assumed constant. In HF communications Watterson's channel model [15] has received the most attention and is recommended by various standards [16]. As illustrated in Fig. 4 the model consists of a tapped-delay line modeling the delays between different propagation paths, which are usually in the order of several milliseconds. The frequency domain characterization is achieved by modulating each of these paths with a random tap gain, corresponding to the varying heights of the ionospheric layers (Doppler shifts) as well as the turbulence within each layer (Doppler spreads).

Watterson has verified by measurement that the tap processes can be accurately modeled by a Gaussian distribution for each of the two magneto-ionic components, which exist in general [15], [16]. In our analysis we will however restrict ourselves to a single Gaussian component with Doppler spread $\sigma_{D}^{2}$ and vanishing Doppler shift $f_{D}=0$ according to the ITU$\mathrm{R}$ recommendation [16].

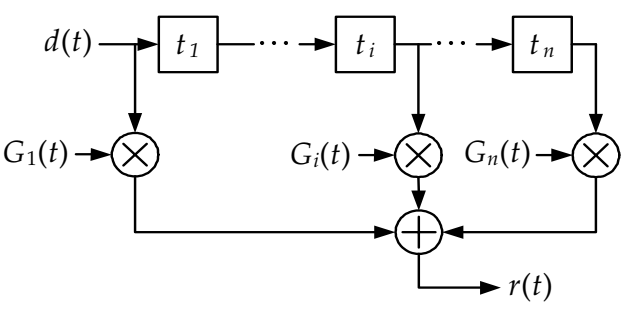

Fig. 4. Watterson's HF-channel model. Delays $t_{i}$ model the multipath propagation, tap processes $G_{i}$ represent the Gaussian power spectral density.

\section{B. Simulation Parameters}

The system parameters are chosen in accordance with the prevalent military standard [11]. In particular, we consider a block transmission structure of alternating pilot and data blocks, a bit-rate of $4.8 \mathrm{kbit} / \mathrm{s}$ and uncoded transmission of 8 PSK symbols. The length of the probe and data blocks is 16 and 32 symbols, respectively. We shall consider the following test cases (cf. Fig. 5).

Plain TR-STBC: This scheme is based on a direct application of TR-STBC as introduced in Sec. II-A. Thus, it makes the false assumption of a constant channel during the transmission of the two subblocks with 32 symbols each.

SB-method: The short-block approach proposed in Sec. II$B$ reduces the size of each subblock to 16 symbols, making the constant channel hypothesis more applicable. IBI occurs between the subblocks since a guard interval is omitted.

MF-method: This method extends the spatio-temporal matched filtering to the variant channel of the second subblock. Thus, the channel is assumed constant over 32 symbols.

SB/MF combination: In addition to the short-block method and the matched-filter technique, we consider a combination of the two. In this case, the length of each subblock is again halved, but instead of considering a constant channel over both blocks, we extend the matched filtering to channels $\mathbf{H}_{1} \rightarrow \mathbf{H}_{3}$ and $\mathbf{H}_{2} \rightarrow \mathbf{H}_{4}$.

Banded Methods: All of the schemes described above assume a constant channel during the transmission of at least one subblock (which has different length for the short-block method and the matched-filter technique, respectively). With increasing Doppler spread this assumption becomes inappropriate for all of the above schemes. Thus, it would be beneficial to model the channel variation on a symbol-to-symbol basis by incorporating this variation into the channel matrices $\mathbf{H}_{1}$ through $\mathbf{H}_{4}$. It has to be noted however that by making this change we forfeit the Toeplitz structure and hence increase computational complexity.

\section{Results}

This section analyzes the performance of our proposed schemes and compares the results to the single antenna case. In terms of BER, we achieve a diversity gain of about 3$4 \mathrm{~dB}$ in $E_{b} / N_{0}$ (not taking the $3 \mathrm{~dB}$ power gain into account). Additionally, we evaluate the performance for varying Doppler spreads and compare the methods' robustness. Finally, we 


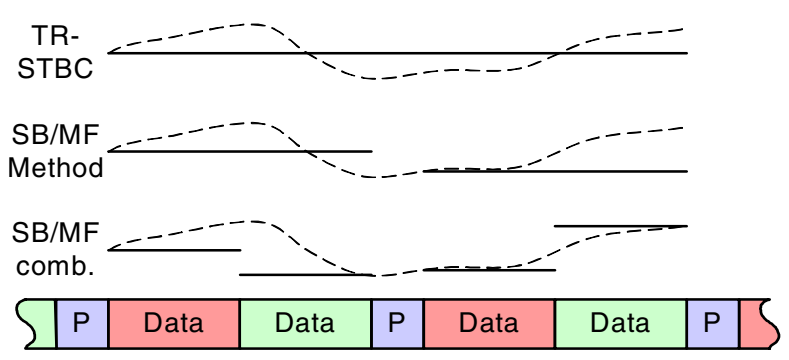

Fig. 5. Approximation of the continuously fading channel at the receiver. The various schemes assume the channel to be constant over different periods of time.

TABLE I

Diversity gain in $E_{b} / N_{0}$ in $\mathrm{dB}$ over the single antenna case at selected values of BER. The values in brackets show the performance loss compared to the banded SB-method.

\begin{tabular}{ccccc}
\hline BER & 0.1 & 0.05 & 0.01 & 0.005 \\
\hline \hline TR-STBC & $0.7(1.4)$ & $0.9(1.7)$ & $0.2(3.4)$ & $-1.1(4.7)$ \\
\hline SB-Method & $2.1(0.0)$ & $2.4(0.1)$ & $2.4(1.0)$ & $2.3(1.3)$ \\
\hline MF-Method eq. & $1.2(1.0)$ & $1.6(1.0)$ & $2.7(0.7)$ & $2.9(0.7)$ \\
\hline SB/MF-Combination & $2.1(0.0)$ & $2.6(0.0)$ & $3.3(0.1)$ & $3.4(0.2)$ \\
\hline SB-Method, banded & 2.1 & 2.6 & 3.4 & 3.6 \\
\hline MF-Method, banded & $1.4(0.7)$ & $2.6(1.0)$ & $2.9(0.5)$ & $3.3(0.3)$ \\
\hline
\end{tabular}

compare the performance of selected block decoding methods.

\section{A. Bit Error Rate vs. $E_{b} / N_{0}$}

In Fig. 6 the BER is plotted versus $E_{b} / N_{0}$ for a fixed delayspread of $2 \mathrm{~ms}$ and a Doppler spread of $0.5 \mathrm{~Hz}$ corresponding to ITU-R's 'poor' channel specification [17]. NDDE has been chosen for block-decoding, since it gives the best performance in all cases. A quantitative comparison is provided in Tab. I.

The results clearly associate a significant performance gain with the methods proposed in this paper. Specifically, they show, that a direct application of TR-STBC performs rather poorly due to its invalid assumptions. The shortblock method shows better performance, but saturates above $E_{b} / N_{0}=5 \mathrm{~dB}$ for two reasons. First, the constant channel hypothesis gradually becomes inappropriate for this method, too. Secondly, interblock-interference has to be suppressed incurring an additional performance penalty. The matchedfilter approach performs quite well in our scenario, however is slightly outperformed by a combination of our short-block and matched-filter technique.

A lower bound for all these methods is achieved when modeling the channel variation on a symbol-by-symbol basis. However, this forfeits the Toeplitz structure of the channel matrices and increases computational complexity. Given the rather small performance gain, this approach may thus not be worthwhile in practice.

\section{B. Bit-Error-Rate vs. Doppler Spread}

In the last example the Doppler spread remained fixed at $\sigma_{d}=0.5 \mathrm{~Hz}$. In order to better understand the different schemes' sensitivity to this parameter, the BER is plotted

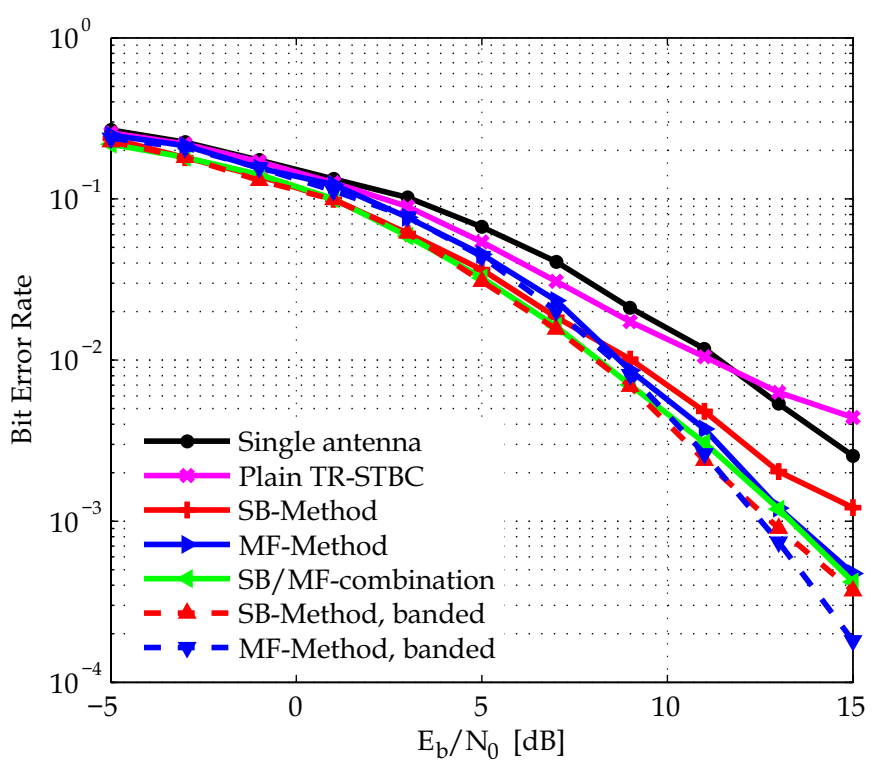

Fig. 6. Bit error rate over $E_{b} / N_{0}$ for the described methods

versus the Doppler spread in Fig. 7. The plot assumes a constant $E_{b} / N_{0}$ of $16 \mathrm{~dB}$.

Several conclusions can be drawn from this comparison. First, at very low Doppler spread the performance of all schemes without IBI converges to conventional TR-STBC, outperforming the remaining techniques. The performance of the shortened-block schemes is slightly worse due to the penalty associated with removing IBI.

As the Doppler spread is increased the constant channel assumption of the different schemes becomes all the less appropriate and thus the performance decreases. It has to be noted that the constant channel hypothesis in the single antenna case has less severe implications (BER remains almost constant as shown in Fig. 7). This can be explained by the fact that there are no cross terms (i.e. subblock $\mathbf{d}_{2}$ influencing the decoding of $\mathbf{d}_{1}$ ) in the decoding process.

\section{Comparison of Block-Decoding Methods}

So far, NDDE has been used for block decoding, since it is giving the best performance. We now analyze how the different methods outlined in Sec. III influence the performance. This is of considerable interest when it comes to a practical implementation since a proper balance between performance and complexity has to be struck.

This comparison is drawn in Fig. 8, for the matched filter approach and the SB/MF combination. In both cases, the block decision feedback algorithms (ZF-BDFE, MMSE-BDFE) outperform their linear counterparts (ZF, MMSE), while NDDE shows the best performance in all cases. However, for the MF-method there is very little difference between the various methods. Thus, in a practical implementation one would be inclined to choose a computationally simpler method and incur the small performance penalty. In contrast, for the SB/MF combination there is a large gap between NDDE and all other 


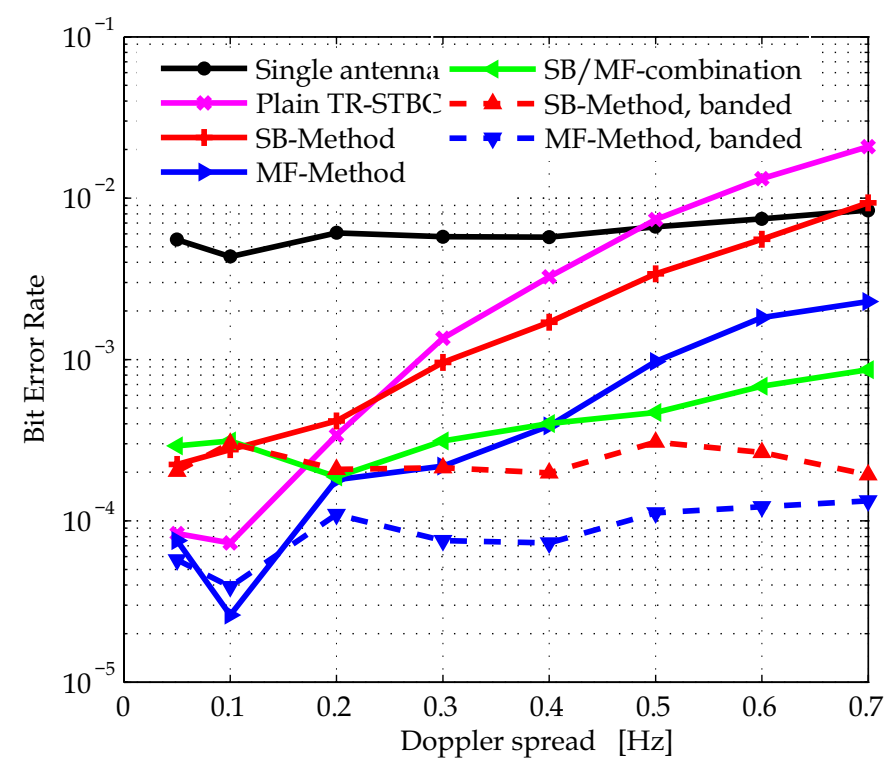

Fig. 7. Bit error rate over Doppler spread for the described methods.
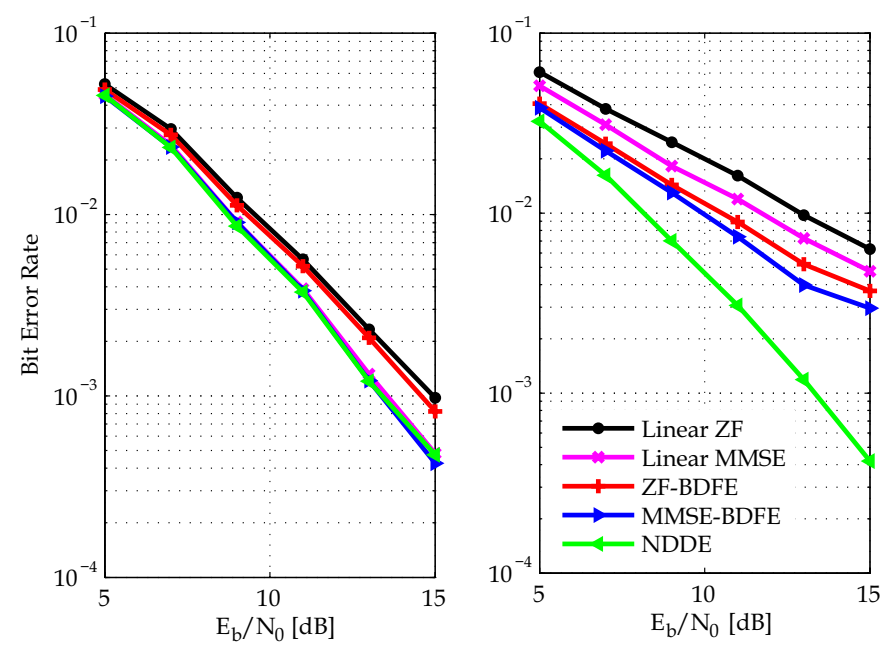

Fig. 8. Block decoding comparison for MF approach (left) and SB/MF combination (right).

methods. The reason for this behavior lies in the IBI. While the decision-feedback schemes are decoding from only one side of the block, NDDE is decoding from both sides. Thus errors made in the IBI region cannot propagate to other parts of the block.

When it comes to a practical implementation it is thus essential to use NDDE when IBI is present in the decoding process. If IBI is avoided by employing guard intervals, then less complex decision feedback schemes might show the better tradeoff between performance and complexity.

\section{Conclusions}

In this paper we proposed two techniques for extending time-reversal space-time block codes to doubly-selective channels and compared their performance for the HF-channel which is a representative example of such a channel. Our results show that the above techniques considerably outperform the single-antenna case by up to $4 \mathrm{~dB}$ (apart from any power gain) for the ITU-R poor channel model. In addition we also examined the schemes' sensitivity to even larger Doppler spreads and compared the performance/complexity tradeoff of various block decoding techniques.

\section{REFERENCES}

[1] S. M. Alamouti, "A Simple Transmit Diversity Technique for Wireless Communications," IEEE J. Select. Areas Commun., vol. 16, no. 8, pp. 1451-1458, Oct. 1998.

[2] E. Lindskog and A. Paulraj, "A Transmit Diversity Scheme for Channels with Intersymbol Interference," in Proc. IEEE International Conference on Communications (ICC), vol. 1, New Orleans, USA, June 2000, pp. 307-311.

[3] Z. Liu, G. Giannakis, A. Scaglione, and S. Barbarossa, "Decoding and equalization of unknown multipath channels based on block precoding and transmit-antenna diversity," in Proc. Thirty-Third Asilomar Conference on Signals, Systems and Computers, vol. 2, Oct. 1999, pp. 15571561.

[4] N. Al-Dhahir, "Single-carrier frequency-domain equalization for spacetime block-coded transmissions over frequency-selective fading channels," IEEE Commun. Lett., vol. 5, no. 7, pp. 304-306, July 2001.

[5] — " "Single-carrier frequency-domain equalization for space-time coded transmissions over broadband wireless channels," in Proc. IEEE International Symposium on Personal, Indoor and Mobile Radio Communciatons, Oct. 2001, pp. B143-B146.

[6] —- "Overview and Comparison of Equalization Schemes for SpaceTime-Coded Signals with Application to EDGE," IEEE Trans. Signal Processing, vol. 50, no. 10, pp. 2477-2488, Oct. 2002.

[7] X. Ma and G. B. Giannakis, "Space-time coding for doubly selective channels," in Proc. International Symposium on Circuits and Systems (ISCAS), vol. 3, May 2002, pp. 647-650.

[8] _ " "Maximum-diversity transmissions over doubly selective wireless channels," IEEE Trans. Inform. Theory, vol. 49, no. 7, pp. 1832-1840, July 2003.

[9] G. B. Giannakis and C. Tepedelenlioglu, "Basis Expansion Models and Diversity Techniques for Blind Identification and Equalization of TimeVarying Channels," Proc. IEEE, vol. 86, no. 10, pp. 1969-1986, Oct. 1998.

[10] B. Sirkeci-Mergen and A. Scaglione, "Randomized distributed spacetime coding for cooperative communication in self organized networks," in Proc. IEEE Workshop on Signal Processing Advances in Wireless Communications (SPAWC), June 2005, pp. 500-504.

[11] Interoperability and Performance Standards for Data Modems, Department of Defense MIL-STD-188-110B, 2000.

[12] I. E. Telatar, "Capacity of Multi-antenna Gaussian Channels," AT\&T Bell Laboratories," Internal Tech. Memo, June 1995.

[13] G. K. Kaleh, "Channel Equalization for Block Transmission Systems," IEEE J. Select. Areas Commun., vol. 13, no. 1, pp. 110-121, Jan. 1995.

[14] F. M. Hsu, "Data Directed Estimation Techniques for Single-Tone HF Modems," in Proc. IEEE Conference on Military Communications (MILCOM), Boston, MA, Oct. 1985, pp. 12.4.1-10.

[15] C. C. Watterson, J. R. Juroshek, and W. D. Bensema, "Experimental Confirmation of an HF Channel Model," IEEE Trans. Commun. Technol., vol. 18, no. 6, pp. 792-803, Dec. 1970.

[16] Testing of HF Modems with Bandwidths of up to $12 \mathrm{kHz}$ Using Ionospheric Channel Simulators, International Telecommunication Union, Radiocommunication Sector (ITU-R) Recommendation F.1487, 2000.

[17] Use of High Frequency Ionospheric Channel Simulators, International Telecommunications Union, Radiocommunications Sector (ITU-R) Rec. F.520-2, 1978. 